\title{
Effect of Noise on the Decoherence of a Central Electron Spin Coupled to an Antiferromagnetic Spin Bath
}

\author{
G. C. Fouokeng, ${ }^{1}$ M. Tchoffo, ${ }^{1}$ S. Moussiliou, ${ }^{2}$ J. C. Ngana Kuetche, ${ }^{1,3}$ \\ Lukong Cornelius Fai, ${ }^{1,4}$ and Massou Siaka ${ }^{2}$ \\ ${ }^{1}$ Laboratory of Mesoscopic and Multilayer Structures, Department of Physics, University of Dschang, Dschang, Cameroon \\ ${ }^{2}$ Departement de Physique, Faculte des Sciences et Technique, Universite d'Abomey-Calavi, 06 BP 48 Cotonou, Benin \\ ${ }^{3}$ Department of Physics, University of Buea, Buea, Cameroon \\ ${ }^{4}$ Department of Physics, Higher Teachers' Training College, University of Bamenda, Bambili, Cameroon
}

Correspondence should be addressed to G. C. Fouokeng; fouokenggc@yahoo.fr

Received 11 October 2013; Revised 21 January 2014; Accepted 21 January 2014; Published 4 March 2014

Academic Editor: Ram N. P. Choudhary

Copyright (C) 2014 G. C. Fouokeng et al. This is an open access article distributed under the Creative Commons Attribution License, which permits unrestricted use, distribution, and reproduction in any medium, provided the original work is properly cited.

\begin{abstract}
We analyze the influence of a two-state autocorrelated noise on the decoherence and on the tunneling Landau-Zener (LZ) transitions during a two-level crossing of a central electron spin (CES) coupled to a one dimensional anisotropic-antiferomagnetic spin, driven by a time-dependent global external magnetic field. The energy splitting of the coupled spin system is found through an approach that computes the noise-averaged frequency. At low magnetic field intensity, the decoherence (or entangled state) of a coupled spin system is dominated by the noise intensity. The effects of the magnetic field pulse and the spin gap antiferromagnetic material used suggest to us that they may be used as tools for the direct observation of the tunneling splitting through the LZ transitions in the sudden limit. We found that the dynamical frequencies display basin-like behavior decay with time, with the birth of entanglement, while the LZ transition probability shows Gaussian shape.
\end{abstract}

\section{Introduction}

The physics of decoherence including LZ transition in a superconducting quantum spin devices is a subject of fundamental importance and of great current interest, for example, in the context of prospective "coupling-CES devices" $[1,2]$. Quantum decoherence remains the most important obstacle to overcome in order to build a quantum computer with these devices. Decoherence due to noise or entanglement with uncontrollable degrees of freedom of the environment is responsible for the decay of the coherent superposition of qubit states. The CES driven by the external noise field has stimulated a lot of interest in the LZ tunneling in periodic structures, although this phenomenon was originally discussed with respect to Bloch oscillations of a crystal of electrons in a strong electric field [3, 4]. Nowadays the most successful experimental systems are semiconductor superlattices [5] that allow access to many different aspects of LZ tunneling $[6,7]$ including decoherence when associated to noise or interaction with the environmental degrees of freedom [8-10]. Thus, it is possible to manipulate the values of the environment or system parameters at any time such that the qubits undergo one-qubit or two-qubit gate operations [11-13].

It is found in the review of many previous works that the decoherence of electron spins coupled to a baths of nuclear spins in quantum dots [14-16] or solid-state impurity centers $[17,18]$ is a key issue in spin-based quantum information processing because of the long coherence time of the quantum spin systems [19-23]. In modern quantum technologies, when the relevant environment is of nanometer size, its quantum nature becomes important [24]. Environments can be modeled as either baths of harmonic oscillators [25] or spins, argued to represent distinct types of environmental modes [26]. The simplest system-environment models consist of a CES coupled to the environment, for example, the spin-boson model [10] that has applications to decoherence of qubits for quantum information processing. 


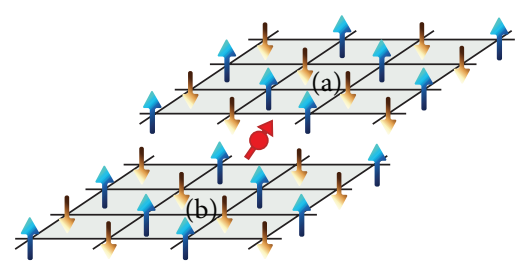

FIGURE 1: Central electron spin coupled to a one-dimensional anisotropic-antiferromagnetic spin systems. The spin configuration is compatible with the spin state in a sublattice (i.e., the spins on each sublattice are antiparallel). (a) and (b) represent physical configuration of a two-interpenetrating quantum spin systems.

Antiferromagnetic superconducting materials have been reported recently to have applications in the area of quantum information processing [27-32].

Therefore in such a system, decoherence of a clusterspin degrees of freedom is expected to arise mainly from the hyperfine coupling with the nuclear spins $[20,21,33]$.

This paper considers a CES coupled to two-interpenetrating spins systems (Figure 1).

Within the framework of dissipative Landau-Zener transitions of qubits [29], we propose a scheme to study decoherence and LZ transition of CES coupled to twointerpenetrating spins systems in state of an antiferromagnetic material with long-range order.

To tackle this problem and tailor the dynamic of the noisy CES coupled spin system, the frequency and the transition probability become figures of merit [21]. The choice of the anisotropic-antiferromagnetic spin Bath makes use of the fact that, considering a two separate sublattices with a certain gap of energy, each sublattice can be expanded in the basis states that are products of single system when coupling with the CES. To solve this problem, we make use of the well-known spin wave approximation (SWA) formalism $[1,2]$.

In [1], the authors investigated the influence of a constant external magnetic field on the parameters characterizing the decoherence of a CES coupled to an antiferromagnetic environment, where the decoherence factor, which displays a Gaussian shape decay with time, depends on the strength of the external magnetic field and on the crystal anisotropy field [1]. This work was extended in our recent paper [2] in which we showed that the two different magnon modes, resulting from the frequency splitting via the application of the variable $\mathrm{B}$-field $(\mathrm{VBF})$, exhibit each a resonance peak of similar amplitude at different time ranges. Taking into account the transition induced by noise in a solid states system [34], we now consider the effect of a noise field which was not considered in works $[1,2]$. This effect is comparable to the two-state autocorrelated (TSAC) noises [35].

The plan of the paper is as follows. In Section 2, we present the model Hamiltonian. Though this problem was studied in our previous article [2], it is important to give a simple analysis of the influence of the TSAC noise on the dynamics of a CES system by evaluating the average frequencies of oscillations. In Section 3 in the light of LZ scenario, the LZ tunneling transition probability is found. We then close the work with the conclusion.

\section{Model Hamiltonian}

Decoherence rates for different superposition vibration states of a spin-1/2 particle at low temperatures in a time VBF may conveniently be modeled by the "CES" model, which couples a central spin $S$ to a spin-bath $B$ of $\mathrm{N}$-antiferromagnetic spin particles in the presence of a TSAC noise [35]. In view of the forgoing argument, we propose the following Hamiltonian:

$$
H=H_{S}+H_{B}+H_{S B}
$$

with

$$
\begin{gathered}
H_{S}=-g \mu_{B} B S_{0}^{z}+\xi(t) S_{0}^{z}, \\
H_{S B}=-\frac{J_{0} S_{0}^{z}}{\sqrt{N}} \sum_{i}\left(S_{a, i}^{z}+S_{b, i}^{z}\right), \\
H_{B}=-g \mu_{B}\left(B+B_{A}\right) \sum_{i} S_{a, i}^{z}-g \mu_{B}\left(B-B_{A}\right) \sum_{j} S_{b, j}^{z} \\
+J \sum_{i, \delta} S_{a, i} S_{b, i+\delta}+J \sum_{j, \delta} S_{b, j} S_{a, j+\delta} \\
+\xi(t)\left(\sum_{i} S_{a, i}^{z}+\sum_{j} S_{b, j}^{z}\right), \\
B=B_{0} \sin \omega t,
\end{gathered}
$$

where $H_{S}$ and $H_{B}$ are, respectively, the Hamiltonian of the CES and of the environment. $H_{S B}$ is the Hamiltonian of the interaction $[1,2,36] . g$ is the gyromagnetic factor and $\mu_{B}$ the Bohr magneton. For simplicity, significant interaction (3) between the CES and the environment is assumed to be of the Ising type (for a more detailed consideration see [37]).

In the presence of the external magnetic field $B$ applied in the $Z$-direction, the spin orientation changes as the direction of the anisotropy field changes. The presence of noise induces fluctuation of the system as well as the spin wave excitations [23]. In [38] the crossover from the case of a single link of the spin system to the bath was analyzed, where the CES is frustrated according to the noise and uniformly coupled to all the spins of the bath. 
In line with the transformations used in $[1,2]$ and considering that the magnetic field frequency is weak (i.e., $\vec{B}=$ $\left.B_{0} \omega t \vec{z}\right)$, the Hamiltonians $H_{S}, H_{S B}$, and $H_{B}$ can be rewritten as $[36,39]$

$$
\begin{gathered}
H(t)=H_{S}+H_{B}^{\prime}+H_{S B}^{\prime}, \\
H_{S}=-\frac{1}{2}(\varepsilon(t)-\xi(t)) \sigma^{z} ; \quad \varepsilon(t)=g \mu_{B} B_{0} \omega t \\
H_{S B}^{\prime}=-\frac{J_{0}}{2 \sqrt{N}} \sum_{k}\left(\beta_{k}^{+} \beta_{k}-\alpha_{k}^{+} \alpha_{k}\right) \sigma^{z} \\
H_{B}^{\prime}=E_{0}^{\prime}+\sum_{k} \omega_{k}^{+}\left(\alpha_{k}^{+} \alpha_{k}+\frac{1}{2}\right) \\
+\sum_{k} \omega_{k}^{-}\left(\beta_{k}^{+} \beta_{k}+\frac{1}{2}\right)
\end{gathered}
$$

where $\alpha_{k}^{+}\left(\alpha_{k}\right)$ and $\beta_{k}^{+}\left(\beta_{k}\right)$ are the creation (annihilation) operators of the two different magnons with wave vector $k$ and frequency $\omega_{k}^{+}\left(\omega_{k}^{-}\right)$, respectively.

Considering the Hamiltonian in (6) and taking the small $k$ approximation, the dynamical frequencies of the coupled spin system are presented as

$$
\begin{aligned}
\omega_{k}^{ \pm}= & 2 M s J \sqrt{\left(\frac{1+g \mu_{B} B_{A}}{2 M S J}\right)^{2}-\frac{1+2 k^{2} l^{2}}{M}} \\
& \pm g \mu_{B} B \mp 2\langle\xi(t)\rangle,
\end{aligned}
$$

where $l$ is the side length of cubic primitive cell of each sublattice and $M$ the number of nearest neighboring atoms. The slow fluctuations of the coupled spin system correspond to low temperatures $T \ll T_{N}$, where $T_{N}$ is the Néel temperature [40]. The frequencies for the two magnon modes plotted in Figures 1 and 2 display a stochastic behavior due to the presence of noise (see (10)).

In analogy to the dressed atom picture [41], the eigenfrequencies $\omega_{k}^{ \pm}$can be associated with dressed states, that is, the oscillator frequencies of state $|\uparrow k\rangle$ and $|\downarrow k\rangle$ in the presence of mutual coupling. There is a characteristic anticrossing with the energy gap due to the frequency splitting of $\Lambda=4\left(\varepsilon_{0}-\right.$ $\langle\xi(t)\rangle)$, where $\varepsilon_{0}$ is the contribution of the Zeeman effect. The splitting increases with the increase of the time VBF and decreases with an increase of the noise intensity.

The entangled state (see Figure 2) is the consequence of decoherence induced in a system. This figure shows also that the noise favors transitions by reducing the bandwidth gap energy level between the two networks. The basin like behavior (see Figure 3) shows how the dispersion relation proceeds with two extreme values corresponding to the high cut-off frequency and the low cut-off frequency. In this work we find that, when the external magnetic field exceeds a critical value $B_{c}$, we have $\omega_{k}^{-}<0$ which indicates that the corresponding branch of magnons is no longer stable due to effects of noise. As a result, we noted that when $\omega_{k}^{-}<0$, the antiferromagnetic polarization flips perpendicular to the field; that is, the magnetic field induces spin flop transition while the presence of noise in the system enhances oscillations.

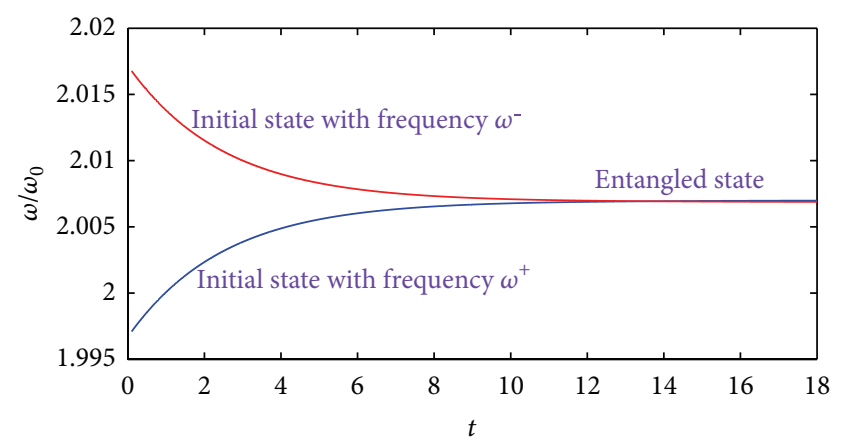

FIgure 2: Plot of the dispersion relation versus time. Red curves for the spin branches with frequency $\omega^{-}$; blue curve for the spin branches with frequency $\omega^{+}$. Both curves entangled at a certain time scales in the presence of noise.

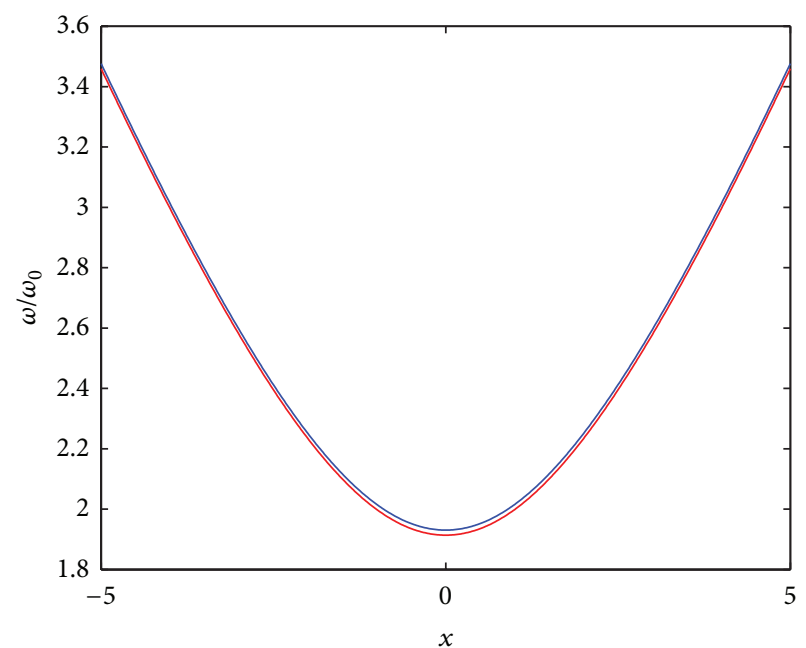

FIGURE 3: Plot of the dispersion relation versus the wave vector $(x=$ $k l$ ). Red curves for the spin branches with frequency $\omega^{-}$; blue curve for the spin branches with frequency $\omega^{+}$. Both curves show basinlike behavior.

\section{Landau-Zener Tunneling Transition Probability}

As in the so-called "tunneling model" of two level system (TLS) defects [42], in the atomic-scale materials defect resides in a potential energy landscape that displays two local minima separated by a small energy gap. For a sufficiently large tunneling matrix element, the eigenstates are appropriate superposition of wave functions localized in the two potential minima.

The characteristic anticrossing occurs in the system with the antiferromagnetic band gap energy due to the frequency splitting or bandwidth frequency $(\hbar=1)$ of $\Lambda=4\left(\varepsilon_{0}-\langle\xi(t)\rangle\right)$, the eigenmodes of the oscillators with frequencies $\omega^{+}, \omega^{-}$ translate the system into a two-level system coupled by a constant magnetic field. In analogy to the case in [43] where the coupling is via a spring constant, whereby in the light of LZ scenario, the frequency difference of the oscillator changes 


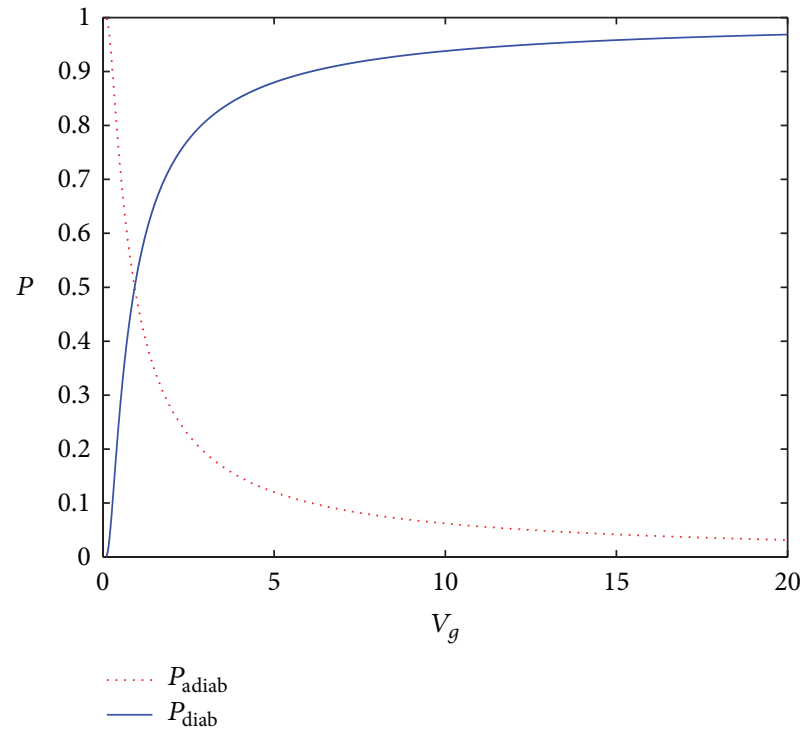

(a)

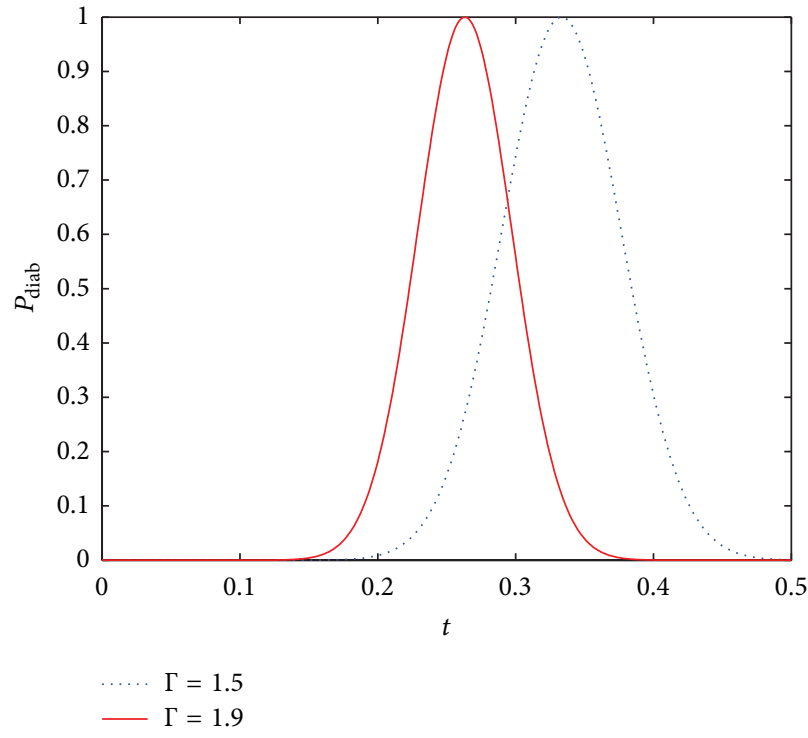

(b)

FIgURE 4: (a) LZ transition probability in the sudden (12) versus the group velocity, for diabatic (dot curves) and for adiabatic cases (solid curve); (b) LZ transition probability versus time for different values of the noise factor $\Gamma$.

linearly in time, the probability for level crossing (diabatic transition) at infinite large time is

$$
P_{\text {diab }}=\exp \left[-\pi \frac{\Lambda^{2}}{2 V_{g}}\right]
$$

Thus attributing this thought to this present issue (6) may be pictured as describing the TLS in which defects exist in both configurational states and corresponds to local minima in the antiferromagnetic potential energy landscape. In the low temperature regime, the dynamics of the system is dominated by the TSAC noise frequency, $\Gamma$. In this case, the incoherent tunneling rate from $|0\rangle$ to $|1\rangle$ is given by the bandwidth frequency $\Lambda$ which changes linearly in time. Thus, with the help of (11), in the consideration of this work, the transition probability for level crossing (diabatic transition) in the sudden limit is

$$
P_{\text {diab }}=\exp \left[-\pi \frac{\left(4\left(\varepsilon_{0}-\langle\xi(t)\rangle\right)\right)^{2}}{2 V_{g}}\right] .
$$

$V_{g}$ is the transition speed given by the dispersion relation $V_{g}=\partial \omega / \partial k$ and $k$ the wave vector. If $a=0$ or $\Gamma \rightarrow+\infty$, then $V_{g} \approx \varepsilon_{0}$, yielding (12) which is exactly the LZ transition probability in a completely coherent sudden limit [44].

In Figures 4(a) to 5(b), considering the stochastic effect of the frequencies due to the TSAC noise, we plot the range of values of time for which the survival probability amplitude is maximum, as it exhibits a resonant peak. There is shrinkage in the width of this resonant peak for large value of the Zeeman energy. This implies that the initially prepared state of the environmental frequency mode could be tailored with precision if certain values of the Zeeman energy $\varepsilon_{0}$, noise amplitude $a$, and noise phase $\Gamma$ are used.
Consequently, Figure 6(a) shows the diabatic survival probability versus the distribution velocity, for different values of the noise phase. In Figure 6(b), the time-dependent survival probability is also plotted. We find that the diabatic probability increases with the increase of the distribution velocity while the noise phase decreases.

As is expected in Figure 5(a), the transition probability plotted as a function of noise phase factor $\Gamma$ shows phase decoherence in the system. The birth of entanglement in the system (see Figure 2) occurs after some time (maximum value) that corresponds to state degeneracy. This time given in (13) is identified as an autocorrelation time (or decoherence time):

$$
\tau=\tau_{r} \ln \left(\frac{a}{\varepsilon_{0}}\right),
$$

where $\tau_{c}$ is a relaxation time. From (13) we find that the long coherence regime of the system corresponds to the high magnetic field intensity and for small value of the noise amplitude $a$.

\section{Conclusion}

In this paper, we have investigated theoretically decoherence and the LZ transition of a superconducting-qubit coupled spin system, where the system exhibit bistability mediated by the CES trapped by the vibronic coupling. As presented in our results, the two magnon modes in the system displayed a stochastic behavior according to (10). It is observed that the environment frequency modes can be tailored with precision by controlling the Zeeman energy, noise amplitude, and noise phase. In the light of LZ scenario, the existence of the energy gap as a tunneling matrix element couples with the Bath 


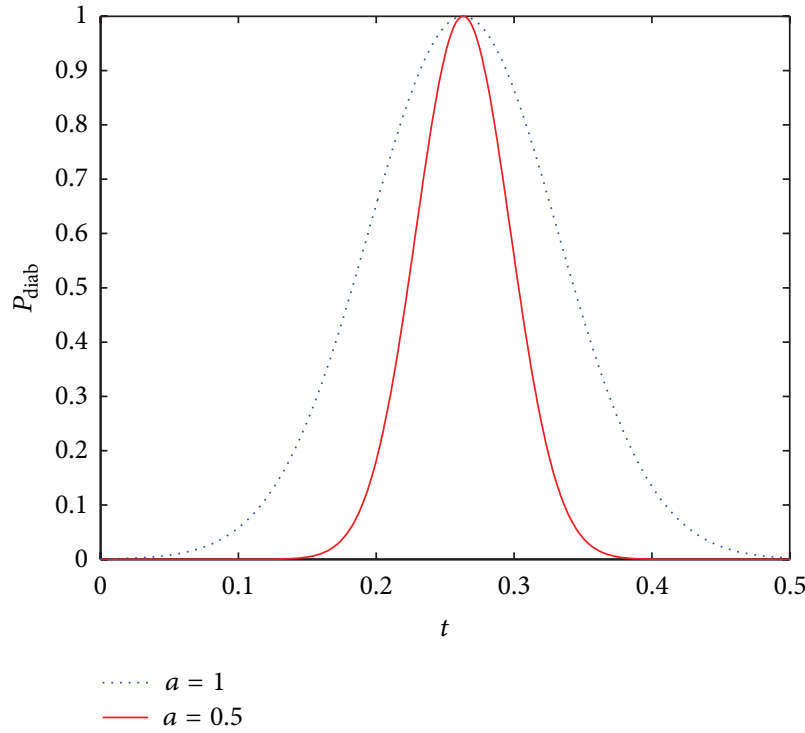

(a)

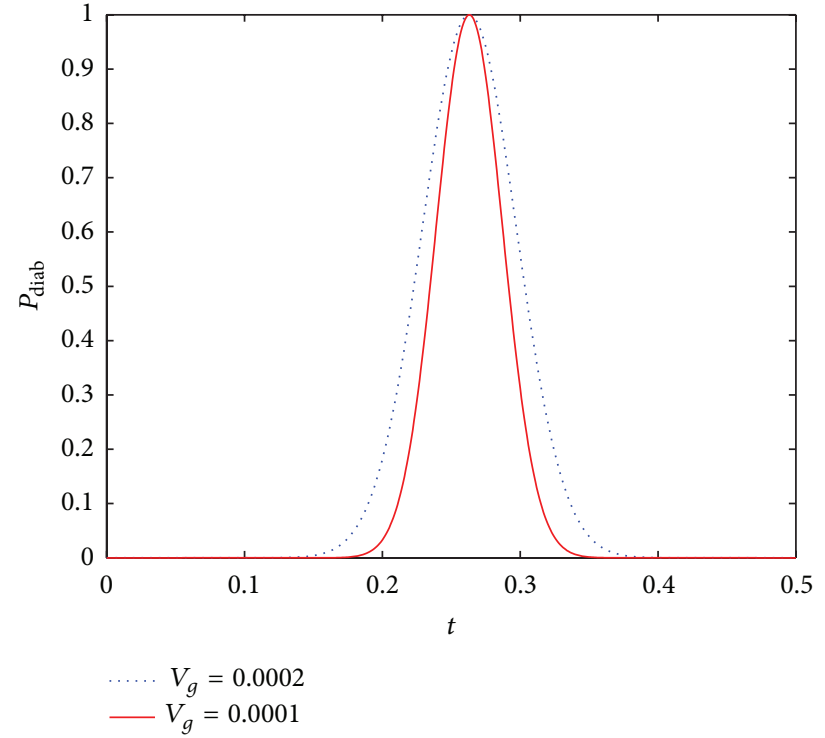

(b)

FIGURE 5: Plots of the LZ transition probability versus time, in the sudden limit expressed in (12). The curves inserted in Figure 5(a) show the behavior for different values of the noise amplitude $a$ and in Figure 5(b) for different values the group velocity.

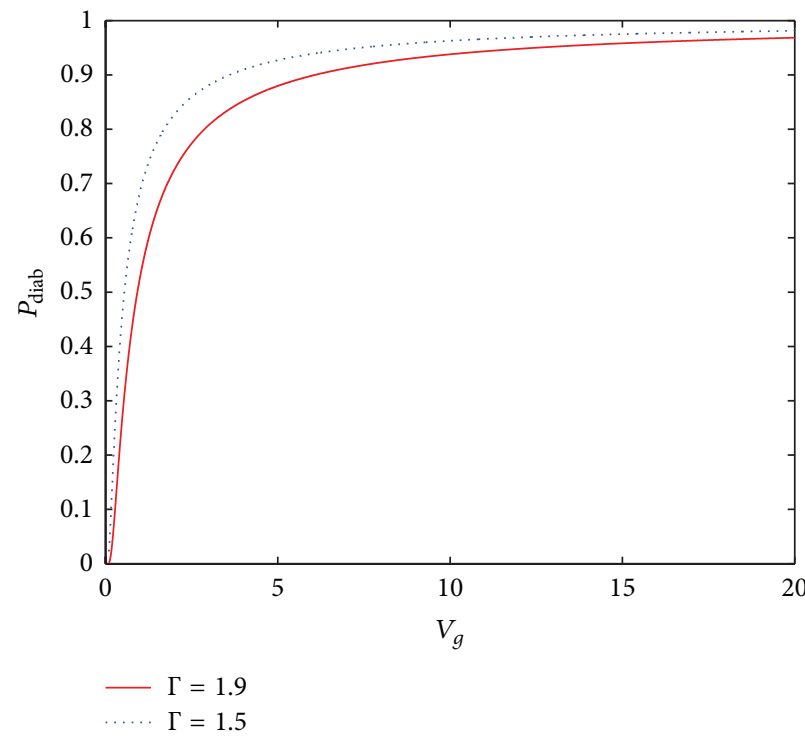

(a)

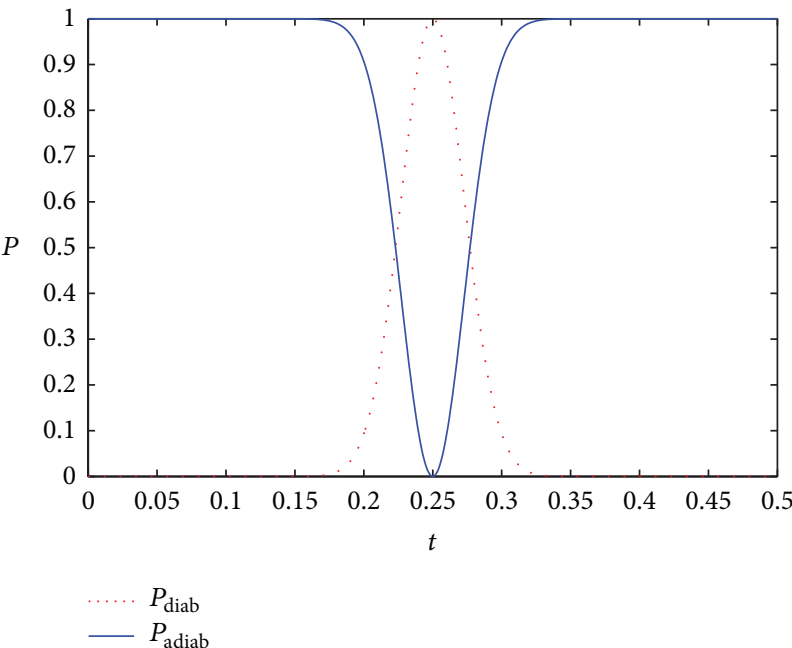

(b)

FIgURE 6: Plots of the LZ transition probability in the sudden limit expressed in (12). Figure 6(a) shows the LZ transition probability in function of the group velocity for different values of the noise factor $\Gamma$ while Figure $6(\mathrm{~b})$ shows the LZ transition probability in function of time in a diabatic (dot curve) and in adiabatic cases (solid curve).

networks; a probability for level crossing occurs. It is shown in this paper that the presence of noise as well as the driving field induces fluctuations in energy levels. These fluctuations modify the transition rate in two ways: (1) due to the TSAC noise, the modifications tends in the direction in which the nonadiabaticity is effectively increased and the probability becomes limited by the upper and the lower bounds. These effects amplify decoherence of the CES. (2) The driving field modifies the transitions in the direction in which the nonadiabaticity decreases and thus this leads to the reduction of decoherence. So, in a high magnetic field regime, the CES is strongly coupled to the spin bath and this contributes to delay decoherence effects in the coupled system. Both dynamical frequencies and LZ transition probability depend on the strength of the time VBF, the noise amplitude, and the crystal anisotropy field. 


\section{Conflict of Interests}

The authors declare that there is no conflict of interests regarding the publishing of this paper.

\section{References}

[1] X.-Z. Yuan, H.-S. Goan, and K.-D. Zhu, "Influence of an external magnetic field on the decoherence of a central spin coupled to an antiferromagnetic environment," New Journal of Physics, vol. 9, article 219, 15 pages, 2007.

[2] M. Tchoffo, G. C. Fouokeng, S. Massou et al., "Effect of the variable B-field on the dynamic of a central electron spin coupled to an anti-ferromagnetic qubit bath," World Journal of Condensed Matter Physics, vol. 2, pp. 246-256, 2012.

[3] F. Bloch and I. I. Rabi, "Atoms in variable magnetic fields," Reviews of Modern Physics, vol. 17, no. 2-3, pp. 237-244, 1945.

[4] L. D. Landau, "On the theory of transfer of energy at collisions II," Physikalische Zeitschrift der Sowjetunion, vol. 2, pp. 46-51, 1932.

[5] R. McDermott, "Materials origins of decoherence in superconducting qubits," IEEE Transactions on Applied Superconductivity, vol. 19, no. 1, pp. 1-13, 2009.

[6] A. Zenesini, C. Sias, H. Lignier et al., "Resonant tunneling of Bose-Einstein condensates in optical lattices," New Journal of Physics, vol. 10, Article ID 053038, 2008.

[7] K. Rapedius, C. Elsen, D. Witthaut, S. Wimberger, and H. J. Korsch, "Nonlinear resonant tunneling of Bose-Einstein condensates in tilted optical lattices," Physical Review A, vol. 82, no. 6, Article ID 063601, 2010.

[8] R. Hanson and D. D. Awschalom, "Coherent manipulation of single spins in semiconductors," Nature, vol. 453, no. 7198, pp. 1043-1049, 2008.

[9] J. Clarke and F. K. Wilhelm, "Superconducting quantum bits," Nature, vol. 453, no. 7198, pp. 1031-1042, 2008.

[10] T. D. Ladd, F. Jelezko, R. Laflamme, Y. Nakamura, C. Monroe, and J. L. O’Brien, “Quantum computers," Nature, vol. 464, no. 7285, pp. 45-53, 2010.

[11] B. Rosam, K. Leo, M. Glück et al., "Lifetime of WannierStark states in semiconductor superlattices under strong Zener tunneling to above-barrier bands," Physical Review B, vol. 68, no. 12, Article ID 125301, pp. 1253011-1253017, 2003.

[12] M. Wubs, K. Saito, S. Kohler, Y. Kayanuma, and P. Hanggi, "Landau-Zener transitions in qubits controlled by electromagnetic fields," New Journal of Physics, vol. 7, article 218, pp. 13672630, 2005.

[13] P. Abumov and D. W. L. Sprung, "Interminiband Rabi oscillations in biased semiconductor superlattices," Physical Review B, vol. 75, no. 16, Article ID 165421, pp. 165421-165433, 2007.

[14] X. Xu, W. Yao et al., "Coupled spin and valley physics in ... of the nuclear field via dark state spectroscopy," Nature, vol. 459, pp. 1105-1109, 2009.

[15] P. Maletinsky, M. Kroner, and A. Imamoglu, "Dynamics of quantum dot nuclear spin polarization controlled by a single electron," Nature Physics, vol. 5, p. 407, 2009.

[16] D. Press, K. De Greve, P. L. McMahon et al., "Ultrafast optical spin echo in a single quantum dot," Nature Photonics, vol. 4, no. 6, pp. 367-370, 2010.

[17] G. Balasubramanian, P. Neumann, D. Twitchen et al., "Ultralong spin coherence time in isotopically engineered diamond," Nature Materials, vol. 8, no. 5, pp. 383-387, 2009.
[18] J. Du, X. Rong, N. Zhao, Y. Wang, J. Yang, and R. B. Liu, "Preserving electron spin coherence in solids by optimal dynamical decoupling," Nature, vol. 461, no. 7268, pp. 1265-1268, 2009.

[19] P. Huang, X. Kong, N. Zhao et al., "Observation of an anomalous decoherence effect in a quantum bath at room temperature," Nature Communications, vol. 2, no. 1, article 570, 2011.

[20] N. Zhao, J.-L. Zhu, R.-B. Liu, and C. P. Sun, "Quantum noise theory for quantum transport through nanostructures," New Journal of Physics, vol. 13, Article ID 013005, 2011.

[21] W. Yao, R.-B. Liu, and L. J. Sham, "Theory of electron spin decoherence by interacting nuclear spins in a quantum dot," Physical Review B - Condensed Matter and Materials Physics, vol. 74, no. 19, Article ID 195301, 2006.

[22] W. Yang and R.-B. Liu, "Quantum many-body theory of qubit decoherence in a finite-size spin bath," Physical Review B, vol. 78, no. 12, Article ID 129901, 2008.

[23] W. Yao, R.-B. Liu, and L. J. Sham, "Restoring coherence lost to a slow interacting mesoscopic spin bath," Physical Review Letters, vol. 98, Article ID 077602, 2007.

[24] C. A. Ryan, J. S. Hodges, and D. G. Cory, "Robust decoupling techniques to extend quantum coherence in diamond," Physical Review Letters, vol. 105, no. 20, Article ID 200402, 4 pages, 2010.

[25] U. Weiss, Quantum Dissipative Systems, World Scientific, Singapore, 2nd edition, 1999.

[26] N. V. Prokof'ev and P. C. E. Stamp, “Theory of the spin bath," Reports on Progress in Physics, vol. 63, no. 4, pp. 669-726, 2000.

[27] J. M. Taylor, C. M. Marcus, and M. D. Lukin, "Long-lived memory for mesoscopic quantum bits," Physical Review Letters, vol. 90, no. 20, Article ID 206803, 4 pages, 2003.

[28] P. J. Metaxas, R. L. Stamps, J.-P. Jamet, J. Ferré, V. Baltz, and B. Rodmacq, "Expansion and relaxation of magnetic mirror domains in a $\mathrm{Pt} / \mathrm{Co} / \mathrm{Pt} / \mathrm{Co} / \mathrm{Pt}$ multilayer with antiferromagnetic interlayer coupling," Journal of Physics Condensed Matter, vol. 24, no. 2, Article ID 024212, 5 pages, 2012.

[29] K. Saito, M. Wubs, S. Kohler, Y. Kayanuma, and P. Hänggi, "Dissipative Landau-Zener transitions of a qubit: Bath-specific and universal behavior," Physical Review B, vol. 75, Article ID 214308, 14 pages, 2007.

[30] S. Kohler, P. Hänggi, and M. WUBS, Path Integrals: New Trends and Perspectives, World Scientific, Singapore, 2008, edited by, W. Janke and A. Pelster.

[31] B.-Q. Jin and V. E. Korepin, "Localizable entanglement in antiferromagnetic spin chains," Physical Review A, vol. 69, no. 6, Article ID 062314, 9 pages, 2004.

[32] F. Meier and D. Loss, "Electron and nuclear spin dynamics in antiferromagnetic molecular rings," Physical Review Letters, vol. 86, no. 23, pp. 5373-5376, 2001.

[33] F. Troiani, A. Ghirri, M. Affronte et al., "Molecular engineering of antiferromagnetic rings for quantum computation," Physical Review Letters, vol. 94, no. 20, 2005.

[34] J. Stehlik, Y. Dovzhenko, J. R. Petta et al., "Landau-ZenerStuckelberg interferometry of a single electron charge qubit," Physical Review B, vol. 86, Article ID 121303, 5 pages, 2012.

[35] P. Hanggi and P. Jung, "colored noise in dynamical systems," Advance in Chemical Physics, vol. 39, p. 239, 1995.

[36] C. Kittel, Quantum Theory of Solids, Wiley, NewYork, NY, USA, 1963.

[37] M. Baenninger, A. Ghosh, M. Pepper et al., "Local transport in a disorder-stabilized correlated insulating phase," Physical Review B, vol. 72, no. 24, Article ID 241311, 2005. 
[38] D. Rossini, T. Calarco, V. Giovannetti, S. Montangero, and R. Fazio, "Decoherence induced by interacting quantum spin baths," Physical Review A, vol. 75, no. 3, Article ID 032333, 2007.

[39] O. Madelung, Introduction to Solid-State Theory, vol. 2 of SolidState Sciences, Springer, Berlin, Germany, 1978.

[40] K. Yosida, Theory of Magnetism, vol. 122 of Solid-State Sciences, Springer, Berlin, Germany, 1996.

[41] C. Cohen-Tannoudji, J. Dupont-Roc, and G. Grynberg, AtomPhoton Interactions, Wiley-VCH, Weinheim, Germany, 2004.

[42] W. A. Phillips, Ed Amorphous Solids: Low-Temperature Properties, Springer, New York, NY, USA, 1981.

[43] L. Novotny, "Strong coupling, energy splitting, and level crossings: a classical perspective," American Journal of Physics, vol. 78, no. 11, pp. 1199-1202, 2010.

[44] P. Ao and J. Rammer, "Quantum dynamics of a two-state system in a dissipative environment," Physical Review B, vol. 43, no. 7, pp. 5397-5418, 1991. 

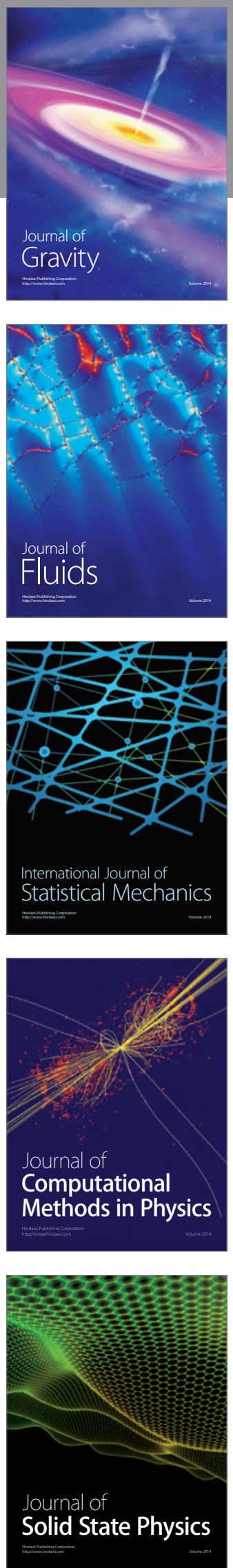

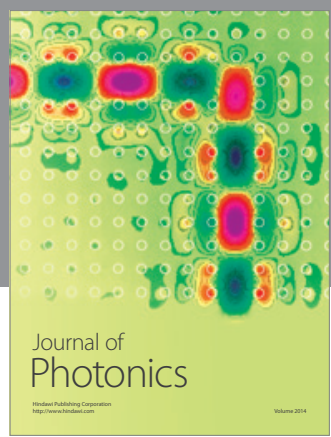

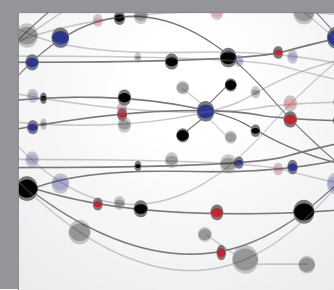

The Scientific World Journal

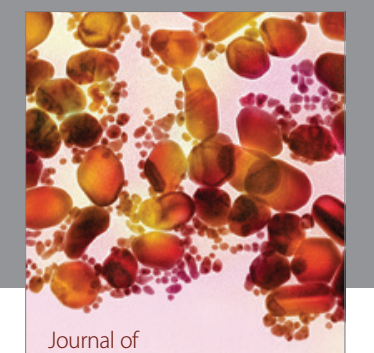

Soft Matter
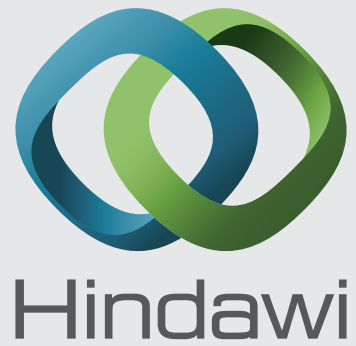

Submit your manuscripts at

http://www.hindawi.com
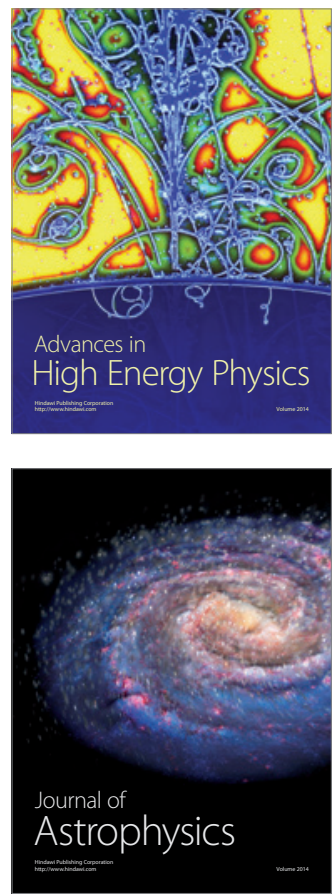
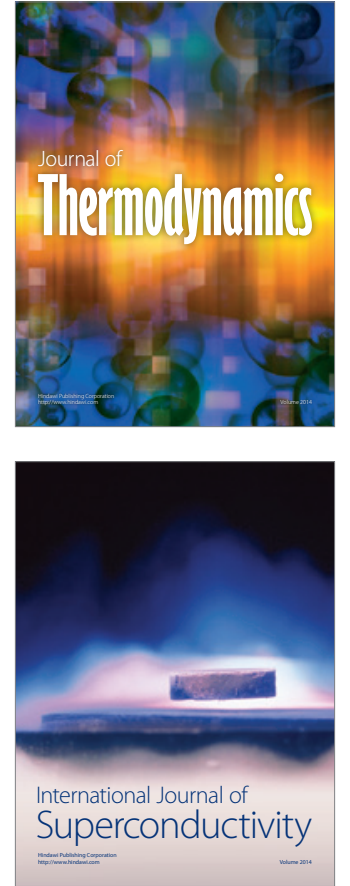
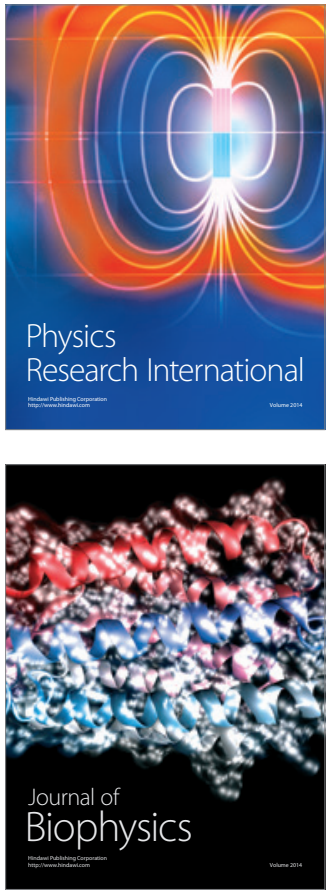
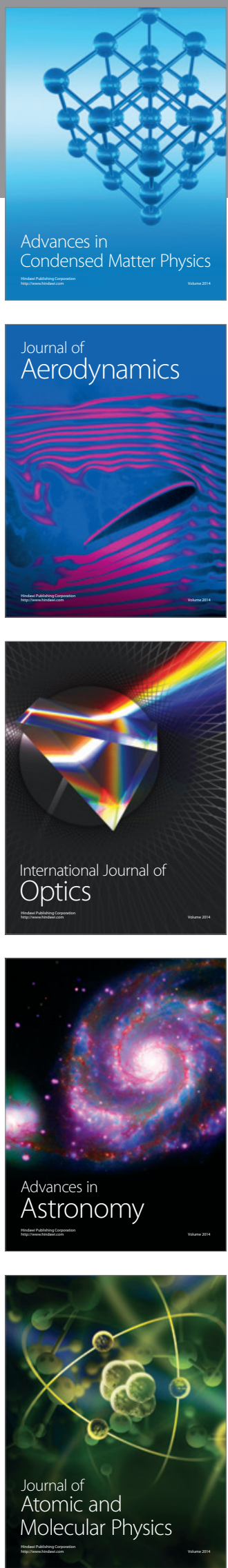